\title{
SPACE FORMS FROM THE VIEWPOINT OF THEIR GEODESIC SPHERES
}

\author{
TOSHIAKI AdAchi AND SadahIRo MaEda
}

In this paper, we focus our attention on the extrinsic shape of geodesics on geodesic spheres in a given Riemannian manifold $M$. We characterise real and complex space forms in the class of smooth Riemannian manifolds from this point of view.

\section{INTRODUCTION}

The aim of this paper is to characterise real and complex space forms by observing the extrinsic shape of geodesics on their geodesic spheres.

Real space forms are Riemannian manifolds of constant curvature, which are locally isometric to either one of the standard spheres, Euclidean spaces and real hyperbolic spaces. In a real space form $M$, a geodesic sphere $G_{m}(r)=\{p \in M \mid d(p, m)=r\}$ with centre $m$ and radius $r$ is a totally umbilic but not totally geodesic hypersurface (with parallel second fundamental form). Here, $d$ denotes the distance function induced by the Riemannian metric $\langle$,$\rangle on M$. This fact tells us that every geodesic on $G_{m}(r)$ is a circle of positive curvature in the ambient manifold $M$.

Next we consider geodesic spheres in complex space forms. Complex space forms are Kähler manifolds of constant holomorphic sectional curvature. It is well-known that these are locally complex analytically isometric to one of complex projective spaces, complex Euclidean spaces and complex hyperbolic spaces. We note that a complex space form $M$ which is not locally isometric to a complex Euclidean space does not admit real hypersurfaces all of whose geodesics are circles in $M$. However geodesic spheres are nice examples of real hypersurfaces in $M$. For example, for a geodesic sphere $G_{m}(r)$ of sufficiently small radius $r$, we know that all geodesics orthogonal to $\xi$ on $G_{m}(r)$ and all integral curves of $\xi$ are circles of positive curvature in $M$, where $\xi$ is the characteristic vector field of $G_{m}(r)$ in $M$ (for details, see $[1,5]$ ). In this context we shall give some characterisations of real and complex space forms.

In this paper we study Riemannian manifolds without boundary.

Received 27th October, 1999

The first author partially supported by Grant-in-Aid for Scientific Research (C) (No. 11640073), Ministry of Education, Science, Sport and Culture.

The second author partially supported by Grant-in-Aid for Scientific Research (C) (No. 11640079), Ministry of Education, Science, Sport and Culture.

Copyright Clearance Centre, Inc. Serial-fee code: 0004-9727/00 \$A2.00+0.00. 


\section{EXPANSION FOR THE SECOND FUNDAMENTAL FORM}

Our paper is based on the expansion for the second fundamental form of geodesic spheres due to Chen and Vanheche [3]. For the reader's convenience, we state their result.

Let $M$ be a Riemannian manifold of dimension greater than 2 with Riemannian metric 〈, $\rangle$. We denote by $G_{m}(r)$ a geodesic sphere with centre $m$ and radius $r$ in $M$, and by $A_{m, r}$ the shape operator of $G_{m}(r)$ in $M$ with respect to the outward unit normal vector field. We adopt the following signature of the Riemannian curvature tensor $R ; R(X, Y) Z=\nabla_{[X, Y]} Z-\left[\nabla_{X}, \nabla_{Y}\right] Z$, where $\nabla$ is the Riemannian connection.

LEMMA . [3, Theorem 3.1] For non-zero tangent vectors $v, w \in T_{m} M$ at a point $m \in M$, we choose a unit tangent vector $u \in T_{m} M$ orthogonal to both $v$ and $w$. We denote by $v_{\boldsymbol{r}}, w_{\mathbf{r}} \in T_{\exp _{m}(\boldsymbol{r u})} M$ the parallel displacements of $v, w$ along the geodesic segment $\exp _{m}(s u), 0 \leqslant s \leqslant r$. Then for sufficiently small $r$ we have

$$
\left\langle A_{m, r} v_{r}, w_{r}\right\rangle=\frac{1}{r}\langle v, w\rangle-\frac{r}{3}\langle R(u, v) w, u\rangle+O\left(r^{2}\right) .
$$

\section{Characterisations of Real SPACE FORMS}

In this section we characterise real space forms in terms of the extrinsic shape of geodesics on geodesic spheres.

A smooth curve $\gamma(s)$ in a Riemannian manifold $M$ parametrised by its arclength $s$ is called a circle of curvature $\kappa(\geqslant 0)$, if there exists a field of unit vectors $Y_{s}$ along this curve which satisfies the differential equations: $\nabla_{\dot{\gamma}} \dot{\gamma}=\kappa Y_{s}, \nabla_{\dot{\gamma}} Y_{s}=-\kappa \dot{\gamma}$, where $\kappa$ is a constant and $\nabla_{\dot{\gamma}}$ denotes the covariant differentiation along $\gamma$ with respect to $\nabla$. A circle of null curvature is nothing but a geodesic.

As we mentioned in the Introduction, for a real space form, we know that every geodesic on each geodesic sphere is a circle of positive curvature. We here show the converse holds.

TheOREM 1A. Let $M$ be a Riemannian manifold with $\operatorname{dim} M \geqslant 3$. Then the following conditions are equivalent.

(1) $M$ is a real space form.

(2) At any point $m \in M$, every geodesic on a geodesic sphere $G_{m}(r)$ of $M$ is a circle of positive curvature in $M$ for each sufficiently small $r$.

(3) At any point $m \in M$, every geodesic sphere $G_{m}(r)$ of $M$ is totally umbilic in $M$ for each sufficiently small $r$.

ProOF: $(1) \Rightarrow(2)$. See Introduction.

$(2) \Rightarrow(3)$. Let $\gamma$ be a geodesic on a geodesic sphere $G_{m}(r)$ of sufficiently small radius $r$. We denote by $\nabla$ and $\tilde{\nabla}$ the Riemannian connections of $G_{m}(r)$ and $M$, 
respectively. By the Gauss formula we have

$$
\tilde{\nabla}_{\dot{\gamma}} \dot{\gamma}=\nabla_{\dot{\gamma}} \dot{\gamma}+\left\langle A_{m, r} \dot{\gamma}, \dot{\gamma}\right\rangle N=\left\langle A_{m, r} \dot{\gamma}, \dot{\gamma}\right\rangle N
$$

where $N$ is a unit normal vector field of $G_{m}(r)$ in $M$. Hence the Weingarten formula $\widetilde{\nabla}_{X} N=-A_{m, r} X$ implies

$$
\widetilde{\nabla}_{\dot{\gamma}}\left(\tilde{\nabla}_{\dot{\gamma}} \dot{\gamma}\right)=\left\langle\left(\nabla_{\dot{\gamma}} A_{m, r}\right) \dot{\gamma}, \dot{\gamma}\right\rangle N-\left\langle A_{m, r} \dot{\gamma}, \dot{\gamma}\right\rangle A_{m, r} \dot{\gamma}
$$

On the other hand, since $\gamma$ is a circle in $M$ by hypothesis, there exists a positive constant $\kappa_{\gamma}$ satisfying that

$$
\widetilde{\nabla}_{\dot{\gamma}}\left(\widetilde{\nabla}_{\dot{\gamma}} \dot{\gamma}\right)=-\kappa_{\gamma}^{2} \dot{\gamma}
$$

By comparing the tangential components of (2.1) and (2.2), we find the following:

$$
\left\langle A_{m, r} \dot{\gamma}, \dot{\gamma}\right\rangle A_{m, r} \dot{\gamma}=\kappa_{\gamma}^{2} \dot{\gamma}
$$

In particular, we have for every unit tangent vector $v \in T\left(G_{m}(r)\right)$

$$
A_{m, r} v=\kappa_{v} v \quad \text { or } \quad A_{m, r} v=-\kappa_{v} v
$$

for some positive $\kappa_{v}$. This guarantees that at an arbitrary point $p \in G_{m}(r)$, the equality $A_{m, r} v=k v$ holds with some positive $k$ for each tangent vector $v \in T_{p}\left(G_{m}(r)\right)$ at $p$, that is, $G_{m}(r)$ is umbilic at $p$ in $M$. Hence $G_{m}(r)$ is totally umbilic in the ambient manifold $M$.

(3) $\Rightarrow(1)$. This is a result of Chen and Vanheche (see [3, Theorem 3.3]). For completion we here recall their proof. At an arbitrary point $m \in M$, since $G_{m}(r)$ is totally umbilic in $M$ for sufficiently small $r$, Equation (1.1) yields that

$$
\langle R(u, v) w, u\rangle=0
$$

for any orthonormal vectors $u, v, w \in T_{m} M$. Therefore we obtain the conclusion (see [4]).

Motivated by the argument in the proof of Theorem 1a, we establish the following which is an improvement of Theorem 1a. By the same discussion as in [5] we find

Theorem 1B. A Riemannian manifold $M$ with $\operatorname{dim} M=n \geqslant 3$ is a real space form if and only if at any point $m \in M$ for each sufficiently small geodesic sphere $G_{m}(r)$ of $M$, there exist orthonormal vectors $v_{1}, v_{2}, \ldots, v_{n-1}$ at each point $p$ of $G_{m}(r)$ such 
that all geodesics of $G_{m}(r)$ through $p$ in the direction $v_{i}+v_{j}(1 \leqslant i \leqslant j \leqslant n-1)$ are circles of positive curvature in the ambient manifold $M$.

ProOF: We have only to show that $G_{m}(r)$ is umbilic at a fixed point $p \in G_{m}(r)$ in the ambient manifold $M$. Let $\gamma_{i}=\gamma_{i}(s)(1 \leqslant i \leqslant n-1)$ be geodesics of $M$ with $\gamma_{i}(0)=p$ and $\dot{\gamma}_{i}(0)=v_{i}$. Then the same discussion as in the proof of Theorem 1a yields

$$
\left\langle A_{m, r} v_{i}, v_{i}\right\rangle A_{m, r} v_{i}=\kappa_{i}^{2} v_{i} \quad \text { for } 1 \leqslant i \leqslant n-1
$$

for some positive constants $\kappa_{i}$. Hence we find

$$
\left\langle A_{m, r} v_{i}, v_{j}\right\rangle=0 \quad \text { for } 1 \leqslant i<j \leqslant n-1 .
$$

Let $\gamma_{i j}=\gamma_{i j}(s)(1 \leqslant i<j \leqslant n-1)$ be geodesics of $G_{m}(r)$ with $\gamma_{i j}(0)=p$ and $\dot{\gamma}_{i j}(0)=\left(v_{i}+v_{j}\right) / \sqrt{2}$. So, from (2.4) we similarly get

$$
\left\langle A_{m, r}\left(v_{i}+v_{j}\right),\left(v_{i}+v_{j}\right)\right\rangle A_{m, r}\left(v_{i}+v_{j}\right)=2 \kappa_{i j}^{2}\left(v_{i}+v_{j}\right)
$$

for some positive $\kappa_{i j}$. This implies

$$
\left\langle A_{m, r}\left(v_{i}+v_{j}\right), v_{i}-v_{j}\right\rangle=0 \text { for } 1 \leqslant i<j \leqslant n-1
$$

so that

$$
\left\langle A_{m, r} v_{i}, v_{i}\right\rangle=\left\langle A_{m, r} v_{j}, v_{j}\right\rangle \text { for } 1 \leqslant i, j \leqslant n-1 \text {. }
$$

It follows from (2.5) and (2.6) that $G_{m}(r)$ is umbilic at the fixed point $p \in G_{m}(r)$ in $M$. Therefore we obtain the conclusion.

\section{Characterisations of Complex SPACE FORMS}

In this section we study geodesic spheres in a Kähler manifold $M$ with complex structure $J$. Let $N$ be a unit normal vector field on $G_{m}(r)$. Since $G_{m}(r)$ is a real hypersurface in $M$, it admits an almost contact metric structure $(\phi, \xi, \eta,\langle\rangle$,$) induced$ from the Kähler structure $J$ of $M$, which satisfies

$$
\phi^{2}=-I+\eta \otimes \xi, \quad \xi=-J N, \quad \eta(\xi)=1
$$

(for details, see [2]). We easily have

$$
\begin{aligned}
\left(\nabla_{X} \phi\right) Y & =\eta(Y) A_{m, r} X-\left\langle A_{m, r} X, Y\right\rangle \xi \\
\nabla_{X} \xi & =\phi A_{m, r} X
\end{aligned}
$$

By paying attention on the characteristic vector field $\xi$ of sufficiently small geodesic spheres, we obtain the following characterisation of complex space forms in the class of Kähler manifolds. 
THEOREM 2. Let $M$ be a complex $n(\geqslant 2)$-dimensional Kähler manifold. Then the following conditions are equivalent each other.

(1) $M$ is a complex space form.

(2) At any point $m \in M$, each sufficiently small geodesic sphere $G_{m}(r)$ of $M$ is a Hopf hypersurface of $M$, that is, the characteristic vector $\xi$ of $G_{m}(r)$ is a principal curvature vector in $M$ at each point $p \in G_{m}(r)$.

(3) At any point $m \in M$, for each sufficiently small geodesic sphere $G_{m}(r)$, every integral curve of the vector field $\xi$ is a geodesic on $G_{m}(r)$.

(4) At any point $m \in M$, for each sufficiently small geodesic sphere $G_{m}(r)$, the geodesic on $G_{m}(r)$ through $p$ in the direction of the vector $\xi$ is a circle of positive curvature in $M$ at every point $p \in G_{m}(r)$.

Proof: $(2) \Leftrightarrow(3)$. See Equality (3.2).

(1) $\Rightarrow(2),(1) \Rightarrow(4)$. See $[1,5]$.

(2) $\Rightarrow(1)$. In the Lemma in Section 1 we choose $w$ orthogonal to both $v$ and $J v$ and we set $u=J v$. Since $u_{r}$ is a normal vector of $G_{m}(r)$ in $M$ at $\exp _{m}(r u)$, the vector $v_{r}$ is the characteristic vector of $G_{m}(r)$ at this point. By condition (2), Equation (1.1) shows that the curvature tensor $R$ of $M$ satisfies

$$
\langle R(u, J u) w, u\rangle=0 .
$$

Hence we find that $R(u, J u) u$ is proportional to $J u$ for every $u \in T_{m} M$, so that $M$ is a complex space form (see [6]).

(4) $\Rightarrow(2)$. For a fixed point $p$ of $G_{m}(r)$, we denote by $\gamma$ the geodesic on $G_{m}(r)$ with $\gamma(0)=p$ and $\dot{\gamma}(0)=\xi$. Then by the same discussion as in the proof of Theorem 1a we find that $\tilde{\nabla}_{\dot{\gamma}} \dot{\gamma}=\left\langle A_{m, r} \dot{\gamma}, \dot{\gamma}\right\rangle N$. This, together with the hypothesis that the curve $\gamma$ is a circle in the ambient manifold $M$, implies that $\left\langle A_{m, r} \dot{\gamma}, \dot{\gamma}\right\rangle$ is constant and $\tilde{\nabla}_{\dot{\gamma}} N$ is proportional to $\dot{\gamma}$ for all $s$. Then the Weingarten formula $\widetilde{\nabla}_{X} N=-A_{m, r} X$ shows that $\dot{\gamma}(s)$ is a principal curvature vector for $-\infty<s<\infty$, so that in particular at $s=0$, the vector $\dot{\gamma}(0)=\xi$ is principal. Hence we can see that our geodesic sphere $G_{m}(r)$ is a Hopf hypersurface.

Next we consider the extrinsic shape of geodesics on a geodesic sphere orthogonal to the characteristic vector. The following is an analogy with Theorem la in some sense.

Theorem 3A. A complex $n(\geqslant 2)$-dimensional Kähler manifold $M$ is a complex space form if and only if at an arbitrary point $m \in M$ for any geodesic sphere $G_{m}(r)$ of sufficiently small radius $r$, every geodesic through any fixed point $p$ of $G_{m}(r)$, which is orthogonal to the vector $\xi$ at the point $p$, is a circle of positive curvature in the ambient manifold $M$.

Proof: $(\Longrightarrow)$ See $[1,5]$.

$(\Longleftrightarrow)$ We investigate the shape operator $A_{m, r}$ of a geodesic sphere $G_{m}(r)$ in $M$. For 
a fixed point $p \in G_{m}(r)$ and a fixed unit tangent vector $v \in T_{p}\left(G_{m}(r)\right)$ orthogonal to $\xi$, we denote by $\gamma$ the geodesic with $\gamma(0)=p$ and $\dot{\gamma}(0)=v$. Then the same discussion as in the proof of Theorem 1a yields that there exists some positive $k_{v}$ satisfying

$$
A_{m, r} v=\kappa_{v} v \text { or } \quad A_{m, r} v=-\kappa_{v} v
$$

Hence we see that $A_{m, r} v=k v$ holds with some positive $k$ for any $v \in T_{p}\left(G_{m}(r)\right)$ orthogonal to $\xi$. So, in particular $\xi$ is a principal curvature vector, because $\left\langle A_{m, r} \xi, v\right\rangle=$ $\left\langle\xi, A_{m, r} v\right\rangle=0$ for every tangent vector $v$ orthogonal to $\xi$. Therefore by virtue of Theorem 2 we obtain the conclusion.

As an immediate consequence of our argument we find the following result which corresponds to Theorem $1 \mathrm{~b}$.

TheOREM 3B. A complex $n(\geqslant 2)$-dimensional Kähler manifold $M$ is a complex space form if and only if at an arbitrary point $m \in M$ for any geodesic sphere $G_{m}(r)$ of sufficiently small radius $r$, there exist orthonormal vectors $v_{1}, v_{2}, \ldots, v_{2 n-2}$ orthogonal to the characteristic vector $\xi$ at each point $p$ of $G_{m}(r)$ such that all geodesics on $G_{m}(r)$ through $p$ in the direction $v_{i}+v_{j}(1 \leqslant i \leqslant j \leqslant 2 n-2)$ are circles of positive curvature in the ambient manifold $M$.

\section{REFERENCES}

[1] T. Adachi, S. Maeda and M. Yamagishi, 'Length spectrum of geodesic spheres in a non-flat complex space form', (preprint).

[2] T. Cecil and P. Ryan, 'Focal sets and real hypersurfaces in complex projective space', Trans. Amer. Math. Soc. 269 (1982), 481-499.

[3] B.-y. Chen and L. Vanhecke, 'Differential geometry of geodesic spheres', J. Reine Angew. Math. 325 (1981), 28-67.

[4] A. Fialkow, 'Hypersurfaces of a space of constant curvature', Ann. of Math. 39 (1938), 762-785.

[5] S. Maeda and K. Ogiue, 'Characterizations of geodesic hyperspheres in a complex projective space by observing the extrinsic shape of geodesics', Math. Z. 225 (1997), 537-542.

[6] S. Tanno, 'Constancy of holomorphic sectional curvature in almost Hermitian manifolds', Kodai Math. Sem. Rep. 25 (1973), 190-201.

Department of Mathematics Nagoya Institute of Technology

Gokiso

Nagoya 466-8555

Japan

e-mail: adachi@math.kyy.nitech.ac.jp
Department of Mathematics Shimane University

Matsue

Shimane 690-8504

Japan

e-mail: smaeda@math.shimane-u.ac.jp 